\title{
Personal hygiene among military personnel: developing and testing a self-administered scale
}

\author{
Mohsen Saffari • Harold G. Koenig • \\ Amir H. Pakpour - Hormoz Sanaeinasab • \\ Hojat Rshidi Jahan • Mohammad Gamal Sehlo
}

Received: 19 September 2013/ Accepted: 16 October 2013/Published online: 6 November 2013

(C) The Japanese Society for Hygiene 2013

\begin{abstract}
Objective Good personal hygiene ( $\mathrm{PH})$ behavior is recommended to prevent contagious diseases, and members of military forces may be at high risk for contracting contagious diseases. The aim of this study was to develop and test a new questionnaire on $\mathrm{PH}$ for soldiers.

Methods Participants were all male and from different military settings throughout Iran. Using a five-stage guideline, a panel of experts in the Persian language (Farsi) developed a 21 -item self-administered questionnaire. Face and content validity of the first-draft items were assessed. The questionnaire was then translated and subsequently back-translated into English, and both the Farsi and English versions were tested in pilot studies. The consistency and stability of the questionnaire were tested using Cronbach's alpha and the test-retest strategy. The final scale was administered to a sample of 502 military personnel. Explanatory and confirmatory factor analyses evaluated the
\end{abstract}

\footnotetext{
M. Saffari $(\square)$

Health Research Center, Baqiyatallah University of Medical

Sciences, 1435814783 Tehran, Iran

e-mail: m.saffari@bmsu.ac.ir; Saffari.CHES@gmail.com

M. Saffari - H. Sanaeinasab - H. R. Jahan

Department of Health Education, School of Health, Baqiyatallah

University of Medical Sciences, 1435814783 Tehran, Iran

e-mail: sanain20@yahoo.co.in

H. R. Jahan

e-mail: hojatr73@gmail.com

H. G. Koenig

Department of Psychiatry and Behavioural Sciences, Duke University Medical Center, Durham, NC, USA

e-mail: Harold.Koenig@duke.edu
}

structure of the scale. Both the convergent and discriminative validity of the scale were also determined.

Results Cronbach's alpha coefficients were $>0.85$. Principal component analysis demonstrated a uni-dimensional structure that explained $59 \%$ of the variance in $\mathrm{PH}$ behaviors. Confirmatory factor analysis indicated a good fit (goodness-of-fit index $=0.902$; comparative fitness index $=$ 0.923 ; root mean square error of approximation $=0.0085$ ). Conclusions The results show that this new PH scale has solid psychometric properties for testing $\mathrm{PH}$ behaviors among an Iranian sample of military personnel. We conclude that this scale can be a useful tool for assessing $\mathrm{PH}$ behaviors in military personnel. Further research is needed to determine the scale's value in other countries and cultures.

Keywords Personal hygiene $\cdot$ Validity $\cdot$ Reliability · Scale - Development 


\section{Introduction}

Improvement of personal hygiene (PH) has been an important achievement in public health [1]. Hygiene is defined as those states or activities by which individuals maintain or promote good health through achieving personal cleanness and cleanliness in their surroundings [2]. Hygiene can be separated into two main categories: $\mathrm{PH}$ and environmental hygiene [1]. PH behaviors are the activities of washing/cleaning the body that keep skin, hair, and nails in good condition, of paying attention to oral health and eye and ear care, and of maintaining clean clothes that helps prevents the transmission of contagious diseases [3, 4]. PH includes behaviors such as bathing, hand-washing, dressing, grooming and activities of daily living that maintain cleanliness [5].

Good $\mathrm{PH}$ behavior helps to protect humans against many threats to their physical, social and emotional health and well-being [6]. For example, the transmission of a wide range of parasites, bacteria, and viruses can be prevented by paying attention to $\mathrm{PH}$ [4]. In addition, for those who pay attention to their $\mathrm{PH}$, the risk of social isolation is lower, and the person is more likely to be regarded as a normal and creditable member of society, thereby affecting the emotional and mental health of that individual [7]. Studies show that poor hygiene is often the cause or the result of a range of psychological disorders [8, 9].

Causal relationships have been established between hygienic behaviors and the risk of many infectious illnesses [10]. These diseases account for about one-half of deaths in developing countries and are responsible for nearly $4 \%$ of deaths in developed countries [11]. Some studies indicate that the hygienic behaviors of hand-washing can on its own result in a decrease the incidence of respiratory infections and gastrointestinal disease by 21 and $31 \%$, respectively $[12,13]$.

Knowledge, beliefs, and practices regarding $\mathrm{PH}$ are substantial determinants of future health status and quality of life $[14,15]$. Moreover, these components are related to productivity and performance and have a contributed to the general development of society [13, 16]. Many outbreaks of contagious diseases in recent decades have had a negative impact on community resources [1]. However, research on infectious diseases during the recent past has mainly focused on secondary and tertiary levels of prevention and, unfortunately, primary prevention measures, such as PH, have been overlooked [2].

At the present time, the high frequency of antibiotic prescriptions has caused drug resistance and increased susceptibility to many already controlled infectious diseases [17]. Also, emerging infections, such as acute respiratory syndrome and novel forms of influenza, are presenting new threats to human health [6]. Even in developed countries with strict public health regulations, these types of disorders remain common [1], possibly because exposure to pathogenic microorganisms is an everyday event that can only be avoided by adherence to good PH behaviors [2].

Despite the importance of $\mathrm{PH}$ for individual and social health, there is as yet no widely used measure to evaluate this state systematically. One explanation is the difficulty in measuring $\mathrm{PH}$, which consists of behaviors that may not be readily observable [18]. However, it is important to have valid and reliable instruments to measure PH. Self-reported scales may be useful in this regard [19].

Personal hygiene is relevant to many population groups [7]. People who work and live in the crowded areas may be at particularly high risk for poor hygienic behaviors [10]. Military settings are a prime example of such a high-risk population as military personnel live and work closely together in performing their duties as soldiers [20]. As these individuals receive rigorous training related to their duties, many activities that relate to their health may be ignored [21]. In addition, there are numerous infectious diseases related to respiratory, gastrointestinal, and skin disorders that are easily preventable through attention to proper hygiene $[6,22,23]$. The results of a number of studies indicate that behaviors such as regular brushing of teeth, respiratory etiquette, and hand-washing receive little attention among army personnel [24-26]. Moreover, military personnel often live in environments that are poorly equipped with health facilities and equipment and are often responsible for handling unsanitary substances [21]. Therefore, this population can be exposed to conditions that facilitate the transmission of infectious diseases. The aim of this study was to develop a brief self-rated measure to assess the behaviors of military personnel with regard to their PH.

\section{Materials and methods}

\section{Design and sample}

This was a cross-sectional study in which we developed and tested a new scale to assess PH behaviors in military settings. In total, 594 participants from ten different military settings throughout Iran were included across all stages of the study. The military settings were selected using a simple random sampling method. Participants in each setting were recruited as a sample of convenience. Inclusion criteria were voluntary participation and at least 1 year of experience in the military.

All participants signed an informed consent before entering the study. The survey was approved by institutional review board of the Iranian Department of Military 
Research. The study was conducted during a 2-month period from October to December 2012.

\section{Development of the scale}

The method described by Emami et al. [27] was used to develop and test the scale. This method involved six phases: (1) identify items by a comprehensive literature review and use of experts; (2) assess the face and content validity of items in the initial draft by experts; (3) backward and forward language translation of the scale; (4) assess reliability; (5) evaluate content validity by pilot testing; and (6) develop the final version and use this version in a large target population.

As a first step, a panel of experts was formed. This panel comprised four health education specialists, two nurses with a background in research and in $\mathrm{PH}$, two nurses and one physician with experience caring for soldiers in military clinics, an expert in instrument development, and a statistician. Five members of the panel were bilingual in Farsi and English, and almost all were familiar with the English language. A comprehensive literature review identified three scales related to the topic of the study $(\mathrm{PH})$ $[18,28,29]$, but many PH domains were not contained in these scales. The panel of experts decided to add more items to cover the missing domains, leading ultimately to a total 21 items that covered all of the relevant domains of $\mathrm{PH}$, as agreed to by consensus of the panel.

As a second step, the face validity of the scale was evaluated using the impact score method. As described by Anastasi, this type of validity refers to what the scale seems to measure-not what it factually measures [30]. A number of untrained observers which were part of the target population were asked to examine the scale. To this end, 20 questionnaires were distributed to members of the target group (military personnel) with the request that the recipient rate the importance of each item on a Likert scale ranging from 1 (not at all important) to 5 (very important). This method was used to calculate impact scores for each item by multiplying the percentage of participants that indicated 4 or 5 for an item by the mean score of importance. All items with an impact score of $>1.5$ were retained in the scale [31]. In addition, during a 60-min session involving ten military personnel, the relevancy, ambiguity and difficulty level of each item were discussed. All 21 items were retained, and only minor wording changes were made to improve item clarity.

We next examined content validity using the content validity index (CVI) and content validity ratio (CVR). This was done by sending the draft scale to ten other specialists, including three experts in health education, four in nursing, and three in public health. The CVI based on simplicity, specificity, and clarity of items was assessed by this panel.
A CVI score of $\geq 0.79$ is considered to be acceptable. The CVR, a method initially proposed by Lawshe, evaluates agreement between raters on how essential the items are to the scale. When more than half of the raters indicate that an item is essential, the item is considered to have acceptable content validity. In this study with ten expert raters, a CVR of $\geq 0.62$ was considered to be acceptable [32].

The third step consisted of translating the scale into English by two bilingual researchers and then back-translating the English version into Farsi by two independent translators without previous exposure to the original scale. Agreement between the backward and forward translations was determined. This step is known to assist in international comparisons of scales [27]. Readability of the English version was determined using the Flesch Reading Ease (FRE) method and determining the Flesch-Kincaid Grade (FKG) Level index using Microsoft Word software (Microsoft, Redwood, WA). We used a 100 point measure, with higher scores indicating greater ease in readability (FRE). A score of $>60$ is considered to be acceptable. The FKG indicates the grade reading level for the scale [33].

In the fourth step comprised an assessment of the stability of the scale stability in which 2-week test-retest reliability was determined in a sample of 20 military personnel using the Spearman correlation. Internal consistency was determined by calculating Cronbach's alpha and the item-total score correlation. To further assess content validity of the scale, we also asked 30 military personnel to complete the Farsi version of the scale; the English version was also examined in 12 military personnel who were bilingual in Farsi and English. Comments by these groups were used to further improve the readability and clarity of items on the scale in these two languages.

Finally, the resulting scale was used to assess $\mathrm{PH}$ behaviors in a separate group of 502 military personnel (main sample), with the sample size calculated using Cohen's tables for descriptive studies. The construct validity of the scale was examined using exploratory factor analysis, the principal component method with varimax rotation was utilized to extract factors, the Kaiser-MayerOlkin (KMO) test was used to assess the adequacy of the sample, and the number of components was determined based on the Kaiser-Gutmann rule and on a diagram of the scree plot. Bartlett's test of sphericity examined the multicollinearity of scale items. A confirmatory factor analysis (CFA) was performed to assess the dimensionality of the model, and model fitness was determined using the root mean square error of examination (RMSEA), the comparative fit index (CFI), and the goodness-of-fit index (GFI).

SPSS for Windows ver. 20 and its add-on module AMOS (SPSS Inc., Chicago, IL) were used for the statistical analyses. Mean, standard deviations (SD), percentage, and frequency were used to describe the data. The Student 
$t$ test was used to assess discriminative and convergent validity. The Spearman correlation between the score on the PH scale and health status was computed. All analyses were considered to be statistically significant at a $p \leq 0.05$.

Description of the scale

The PH scale which we have developed and tested is a unique and unprecedented measure to assess PH behaviors considered to be particularly important for preventing infectious diseases. It contains items on 11 main areas of PH that are recommended by the World Health Organization (WHO) and UK Department of Health [4, 34], including oral hygiene (4 items), bathing (3), hand washing ( 2 items), hair care (2 item), nail care (1 item), foot care (1 item), wearing clean clothes (2 item), respiratory hygiene ( 2 item), attention to body odor ( 1 item), eye care ( 2 items), and ear care ( 1 item). These 21 items were selected from a pool of items extracted from existing scales as well as items suggested by our expert panel. Respondents are asked to choose from one of four options ranging from 0 (never) to 3 (always) for each item (see Appendix 1). The total score is arrived at by summing the scores of all 21 items and ranges from 0 to 63 . Higher scores indicate better PH behaviors. There is no recommended cutoff score for the scale at this time.

We also assessed such demographic characteristics as age, marital status, number of children, educational level, background diseases, and sources of information about PH. In addition, participants were asked to rate their current health status on a horizontal line from 0 (very poor) to 100 (very good).

\section{Results}

Participants

All participants were males. Mean age of the main sample $(n=502)$ was 33.04 (SD 6.83). More than $80 \%$ were married. Only $14.8 \%$ had received a post-graduate education. Average time in the military was 13.54 years (SD 6.29). More than $90 \%$ reported no illness at the time of the survey. TV and radio were the most common sources of information on $\mathrm{PH}$. The mean health status score was 86 (SD 14.9), and the average score for the PH scale among participants was 44.17 (SD 10.05). Table 1 provides detailed information on the demographic characteristics of the pilot sample and main sample.

Validity and reliability of PH scale

In the assessment of face validity using the impact score method, no score was $<1.5$ per item, and so all items were
Table 1 Demographic characteristics of pilot and main samples

\begin{tabular}{|c|c|c|}
\hline Demographic variables & $\begin{array}{l}\text { Pilot sample } \\
(n=42), n(\%)\end{array}$ & $\begin{array}{l}\text { Main sample } \\
(n=502), n(\%)\end{array}$ \\
\hline \multicolumn{3}{|l|}{ Age (years) } \\
\hline$<25$ & $8(19.0)$ & $78(15.5)$ \\
\hline $25-35$ & $25(59.5)$ & $286(57.0)$ \\
\hline$>35$ & $9(21.5)$ & $138(27.5)$ \\
\hline \multicolumn{3}{|l|}{ Marital status } \\
\hline Single & $5(11.9)$ & $77(15.3)$ \\
\hline Married & $37(88.1)$ & $425(84.7)$ \\
\hline \multicolumn{3}{|l|}{ Number of children } \\
\hline 0 & $21(50.0)$ & $212(42.2)$ \\
\hline $1-2$ & $18(42.9)$ & $225(44.8)$ \\
\hline $3-4$ & $3(7.1)$ & $65(13.0)$ \\
\hline \multicolumn{3}{|l|}{ Education level } \\
\hline High school & $14(33.3)$ & $202(40.2)$ \\
\hline Graduate & $18(42.9)$ & $226(45.0)$ \\
\hline Post-graduate & $10(23.8)$ & $74(14.8)$ \\
\hline \multicolumn{3}{|c|}{ Military experience (years) } \\
\hline$<10$ & $17(40.5)$ & $125(24.9)$ \\
\hline $10-20$ & $21(50.0)$ & $316(62.9)$ \\
\hline$>20$ & $4(9.5)$ & $61(12.2)$ \\
\hline \multicolumn{3}{|l|}{ Disease } \\
\hline Yes & $2(4.8)$ & $49(9.8)$ \\
\hline No & $40(95.2)$ & $453(90.2)$ \\
\hline \multicolumn{3}{|c|}{ Main information resources } \\
\hline $\mathrm{TV}$ and radio & $25(59.5)$ & $363(72.3)$ \\
\hline Printed material & $12(28.6)$ & $98(19.6)$ \\
\hline Colleagues & $3(7.1)$ & $29(5.8)$ \\
\hline Others & $2(4.8)$ & $12(2.3)$ \\
\hline \multicolumn{3}{|c|}{ Self-rated health status $(0-100)$} \\
\hline$\leq 50$ & $3(7.1)$ & $23(4.6)$ \\
\hline$>50$ & 39 (92.9) & 479 (95.4) \\
\hline
\end{tabular}

included in the PH scale. The CVI and CVR were 0.83 and 0.78 , respectively. Scores on the readability indices for the English version were acceptable (FRE 86.9; FKG 3.7). The scale's Cronbach alpha in step 4 was 0.82 ; the 2-week testretest reliability in this step was 0.88 . Internal consistency was also approved in the main study among the 502 participants $(\alpha=0.89)$. The KMO index (construct validity) was 0.91 , which is acceptable. The Bartlett test of sphericity was significant $(p<0.001)$, and there was no evidence of multicollinearity between items. Both the KaiserGutmann rule and the scree plot showed a single dimension for the scale. The eigenvalue for the first factor was 12.49 , and this one-factor solution explained about $59 \%$ of the variance of the $\mathrm{PH}$ scale score. The values for the communalities of shared variance were $>0.50$. The CFA confirmed a single-factor structure for the $\mathrm{PH}$ scale and 
Table 2 Descriptive analysis, item reliability, and factor loadings of the personal hygiene scale $(n=502)$

\begin{tabular}{llllll}
\hline Items & Mean & $\begin{array}{l}\text { Standard } \\
\text { deviation }\end{array}$ & $\begin{array}{l}\text { Corrected } \\
\text { item total } \\
\text { correlation }\end{array}$ & $\begin{array}{l}\text { Cronbach's } \alpha \\
\text { (if item } \\
\text { deleted) }\end{array}$ & $\begin{array}{l}\text { Factor } \\
\text { loadings }\end{array}$ \\
\hline Q1 & 1.94 & 0.850 & 0.767 & 0.884 & 0.823 \\
Q2 & 2.14 & 0.869 & 0.601 & 0.889 & 0.658 \\
Q3 & 2.44 & 0.811 & 0.710 & 0.886 & 0.772 \\
Q4 & 2.08 & 0.961 & 0.611 & 0.889 & 0.665 \\
Q5 & 1.67 & 0.873 & 0.638 & 0.888 & 0.685 \\
Q6 & 1.34 & 0.924 & 0.554 & 0.891 & 0.597 \\
Q7 & 2.45 & 0.971 & 0.492 & 0.893 & 0.543 \\
Q8 & 2.17 & 0.809 & 0.750 & 0.885 & 0.818 \\
Q9 & 2.50 & 0.703 & 0.715 & 0.886 & 0.784 \\
Q10 & 2.12 & 0.811 & 0.769 & 0.884 & 0.840 \\
Q11 & 2.20 & 0.805 & 0.771 & 0.884 & 0.834 \\
Q12 & 2.25 & 0.825 & 0.846 & 0.882 & 0.916 \\
Q13 & 2.09 & 0.883 & 0.814 & 0.883 & 0.875 \\
Q14 & 1.92 & 0.811 & 0.727 & 0.886 & 0.793 \\
Q15 & 1.97 & 0.913 & 0.651 & 0.888 & 0.712 \\
Q16 & 1.69 & 0.894 & 0.645 & 0.888 & 0.703 \\
Q17 & 2.32 & 0.783 & 0.792 & 0.884 & 0.852 \\
Q18 & 2.21 & 0.831 & 0.792 & 0.884 & 0.851 \\
Q19 & 2.34 & 0.769 & 0.766 & 0.885 & 0.835 \\
Q20 & 2.40 & 0.830 & 0.727 & 0.886 & 0.795 \\
Q21 & 1.94 & 0.939 & 0.696 & 0.887 & 0.729 \\
\hline
\end{tabular}

demonstrated good fit for the model (GFI 0.902; CFI 0.923; RMSEA 0.0085). Descriptive analysis and validity and reliability psychometrics for the $\mathrm{PH}$ scale are presented in Table 2. A significant correlation $(r=0.29, p<0.001)$ was found between the PH scale score and the self-rated health status score, thereby providing some support for convergent validity. Other indicators of convergent and discriminant validity are presented in Table 3 .

\section{Discussion}

Here we describe the development and testing of a new scale to assess PH behaviors in military settings (which might also be useful in other group settings). The results support the validity and reliability of the scale and its single dimension.

The scale was developed using a multi-stage procedure used previously in establishing the psychometric properties of another new scale [27]. Two approaches are suggested when developing a self-rated scale for use in a different culture. First, a new scale may be developed, but it must be assessed using empirical data, and a solid theoretical rationale must exist for the scale's content. A second approach involves translating a pre-existing measure into
Table 3 Discriminative and convergent validity of the personal hygiene scale $(n=502)$

\begin{tabular}{|c|c|c|}
\hline Variables & $\begin{array}{l}\text { Mean } \pm \text { standard } \\
\text { deviation }\end{array}$ & $\begin{array}{l}p \text { value } \\
(t \text { test })\end{array}$ \\
\hline \multicolumn{3}{|l|}{ Age (years) } \\
\hline$\leq 30$ & $43.60 \pm 12.45$ & 0.486 \\
\hline$>30$ & $44.40 \pm 8.93$ & \\
\hline \multicolumn{3}{|l|}{ Marital status } \\
\hline Single & $43.62 \pm 13.59$ & 0.685 \\
\hline Married & $44.28 \pm 9.28$ & \\
\hline \multicolumn{3}{|l|}{ Number of children } \\
\hline$\leq 2$ & $44.10 \pm 10.29$ & 0.679 \\
\hline$>2$ & $44.66 \pm 8.30$ & \\
\hline \multicolumn{3}{|l|}{ Education level } \\
\hline High school & $44.55 \pm 10.86$ & 0.487 \\
\hline Graduate or higher & $43.92 \pm 9.47$ & \\
\hline \multicolumn{3}{|l|}{ Experience (year) } \\
\hline$\leq 15$ & $43.82 \pm 10.29$ & 0.184 \\
\hline$>15$ & $45.19 \pm 9.26$ & \\
\hline \multicolumn{3}{|l|}{ Disease } \\
\hline Yes & $42.09 \pm 11.81$ & 0.043 \\
\hline No & $45.81 \pm 9.85$ & \\
\hline \multicolumn{3}{|c|}{ Main information resources } \\
\hline $\mathrm{TV}$ and radio & $44.51 \pm 9.78$ & 0.227 \\
\hline Others & $43.30 \pm 10.70$ & \\
\hline \multicolumn{3}{|l|}{ Health status $(0-100)$} \\
\hline$\leq 50$ & $42.14 \pm 11.27$ & 0.037 \\
\hline$>50$ & $45.93 \pm 9.68$ & \\
\hline
\end{tabular}

the target language using assistance from specialists [32]. Although the former is difficult and time-consuming work, in our case there was no scale currently available to assess $\mathrm{PH}$ behaviors, forcing us to develop a new scale.

Guidelines have been published to assist researchers in developing new scales. For example, Cotrell and Mckenzie proposed a 14-stage framework for developing new measures in health education and promotion programs [32]. Other researchers have developed a 12-step procedure for developing a new scale for use by healthcare professionals [19]. Our study followed a six-stage protocol for developing the $\mathrm{PH}$ scale, and our results indicate that this approach was successful.

Principal component analysis revealed that the $\mathrm{PH}$ scale is a uni-dimensional scale; this result was further supported by the CFA. Although our PH scale is assessed here using questions that focus on hygienic behaviors with regard to different parts of the body (e.g., mouth, eyes, ears, hands, and feet), these behaviors are correlated with the overall concept of PH [4]. Many studies have found that subjects who pay attention to their $\mathrm{PH}$ in some parts of their body parts are usually attentive to have good $\mathrm{PH}$ in other parts of their body 
as well $[6,8,10]$. Thus, the one-factor structure of the scale also appears to be logical from a theoretical perspective as well.

In terms of the psychometric properties, the Cronbach's alpha in both studies (pilot and main study) were acceptable ( $>0.7)$, thereby supporting the internal consistency of the scale. The high test-retest reliability coefficient also confirms the reliability of the scale. We also assessed scale validity using several methods. Both face validity and content validity were established. Factor analysis also demonstrated a fit structure for the scale. A scale is also expected to have good construct validity, as evidenced by good convergent and discriminant validity, as we demonstrated [32]. We predicted that a person who shows respect for their PH will also have a better health status, which we have now demonstrated using the PH scale. Such relationships have been found in similar studies $[35,36]$.

The PH scale takes only about $10 \mathrm{~min}$ to complete. Tests of scale readability also demonstrated that it was easy to read at the level of a 4th grader. The completion rate of participants in the main study was about $96 \%$ with almost no missing data, supporting the ease of scale administration. We therefore conclude that this scale is brief and easy to complete and that it assesses important dimensions of $\mathrm{PH}$ that most military personnel can identify with.

Overall, respondents in the main study reported moderate levels of PH behaviors, suggesting that such behaviors are generally recognized by military personnel as being important. However, this assessment represents an average, and there were numerous soldiers with either superior or low scores on the scale, indicating need for improvement. These results suggest that educational programs are needed to reinforce the need for military personnel to perform behaviors to maintain their PH. The need for such an educational approach is support by the results of other studies in which some researchers report poor hygienic conditions among military personnel that increase their risk of contracting disease [21, 37]. Two other studies suggest that negative attitudes regarding various $\mathrm{PH}$ behaviors, such as handwashing, are widespread among soldiers [38, 39].

Little hygiene-related research has focused on military personnel, making it difficult to compare the results of our study with those of comparable studies in other populations. Nevertheless, our findings underscore the need for a better understanding of hygienic behaviors in military troops or other occupational fields. Similar research could be conducted in other high-risk populations, such as healthcare professionals, food handlers, or workers in difficult occupations, such as miners or farmers. Assessing such groups using our scale could provide a rationale for developing a program that addresses issues related to the hygiene and self-care for these special groups in order to improve the health of the whole community.
This study has several limitations which must be considered when interpreting the findings. First, this scale was developed and tested only for use in men. However, many females are currently enlisted in military forces, and their hygienic behaviors should also be monitored [20]. Our scale may also be useful in women, but this possibility needs to be established in future studies. Second, although we translated the scale into English, we did not have access to a sufficiently large population of native English speakers in which to evaluate the psychometric properties of the scale. This will need to be established in English-speaking populations in future studies. Third, since no prior scale assessing PH exists, it was not possible to establish the concurrent validity of the scale. However, we used several other methods for establishing the scale's validity here.

In conclusion, our survey of PH behaviors among military and other closed populations is needed to monitor behaviors that are likely to prevent the spread of infectious and other diseases. Assessment tools with solid psychometric properties are needed. Om our study, we developed and tested a new scale for monitoring PH behaviors among military personnel. This scale may be used not only as a monitoring tool in military and other settings for primary prevention, but it may also be used in research studies that seek to establish connections between $\mathrm{PH}$ and the transmission of disease in population groups that work and live closely together. Follow-up studies are then strongly encouraged in different samples and cultures using this psychometrically sound instrument.

Acknowledgments The authors wish to thank Prof. Fazlollah Ahmadi and Prof. Bradley J. Cardinal for their valuable comments on developing the questionnaire. We are also grateful to the Iranian Department of Military Research and Baqiyatallah University of Medical Sciences for their support in conducting the study.

Conflict of interest The authors declare that they have no conflict of interest.

\section{Appendix 1}

We are interested in information about personal hygiene behaviors. Our hope is to identify potential problems in this regard. Please circle one number for each item as it applies to you.

\begin{tabular}{llllll}
\hline No. & Items & Never & Sometimes & Often & Always \\
\hline 1 & $\begin{array}{c}\text { I shower at least once a } \\
\text { day }\end{array}$ & 0 & 1 & 2 & 3 \\
2 & $\begin{array}{c}\text { I comb my hair at least } \\
\text { once a day }\end{array}$ & 0 & 1 & 2 & 3 \\
\hline
\end{tabular}


Appendix continued

\begin{tabular}{|c|c|c|c|c|c|}
\hline No. & Items & Never & Sometimes & Often & Always \\
\hline 3 & $\begin{array}{l}\text { I use soap or shampoo } \\
\text { to clean my body } \\
\text { when showering }\end{array}$ & 0 & 1 & 2 & 3 \\
\hline 4 & $\begin{array}{l}\text { I cut my hair at least } \\
\text { once a month }\end{array}$ & 0 & 1 & 2 & 3 \\
\hline 5 & $\begin{array}{l}\text { I brush my teeth after } \\
\text { each meal }\end{array}$ & 0 & 1 & 2 & 3 \\
\hline 6 & $\begin{array}{l}\text { I use dental floss after } \\
\text { each meal }\end{array}$ & 0 & 1 & 2 & 3 \\
\hline 7 & $\begin{array}{l}\text { I avoid using the } \\
\text { personal hygienic } \\
\text { belongings of others } \\
\text { (e.g., toothbrush, } \\
\text { comb) }\end{array}$ & 0 & 1 & 2 & 3 \\
\hline 8 & $\begin{array}{l}\text { I wash my hands for at } \\
\text { least } 20 \mathrm{~s} \text { with soap } \\
\text { and water before } \\
\text { each meal }\end{array}$ & 0 & 1 & 2 & 3 \\
\hline 9 & $\begin{array}{l}\text { I wash my hands for at } \\
\text { least } 20 \mathrm{~s} \text { with soap } \\
\text { and water after using } \\
\text { the toilet }\end{array}$ & 0 & 1 & 2 & 3 \\
\hline 10 & $\begin{array}{l}\text { I cover my mouth and } \\
\text { nose with a } \\
\text { handkerchief when } \\
\text { sneezing or coughing }\end{array}$ & 0 & 1 & 2 & 3 \\
\hline 11 & $\begin{array}{l}\text { I cut my nails at least } \\
\text { once a week }\end{array}$ & 0 & 1 & 2 & 3 \\
\hline 12 & $\begin{array}{l}\text { I shower after physical } \\
\text { activities or sports } \\
\text { that resulted in } \\
\text { sweating }\end{array}$ & 0 & 1 & 2 & 3 \\
\hline 13 & $\begin{array}{l}\text { I wash my feet at least } \\
\text { once a day }\end{array}$ & 0 & 1 & 2 & 3 \\
\hline 14 & $\begin{array}{l}\text { If I encounter a person } \\
\text { with the signs of a } \\
\text { cold or flu, I will stay } \\
\text { at least } 1-2 \mathrm{~m} \text { away } \\
\text { from her/him }\end{array}$ & 0 & 1 & 2 & 3 \\
\hline 15 & $\begin{array}{l}\text { I use a perfume or } \\
\text { cologne every day }\end{array}$ & 0 & 1 & 2 & 3 \\
\hline 16 & $\begin{array}{l}\text { I change my } \\
\text { underclothes at least } \\
\text { once a day }\end{array}$ & 0 & 1 & 2 & 3 \\
\hline 17 & $\begin{array}{l}\text { I wear clean work } \\
\text { clothes every day }\end{array}$ & 0 & 1 & 2 & 3 \\
\hline 18 & $\begin{array}{l}\text { I brush my teeth at } \\
\text { least } 2-3 \text { min every } \\
\text { time }\end{array}$ & 0 & 1 & 2 & 3 \\
\hline 19 & $\begin{array}{l}\text { I avoid touching my } \\
\text { eyes or mouth with } \\
\text { unclean hands }\end{array}$ & 0 & 1 & 2 & 3 \\
\hline 20 & $\begin{array}{l}\text { I wash my face at least } \\
3 \text { times a day }\end{array}$ & 0 & 1 & 2 & 3 \\
\hline 21 & $\begin{array}{l}\text { I clean my ears at least } \\
\text { once a week }\end{array}$ & 0 & 1 & 2 & 3 \\
\hline
\end{tabular}

\section{References}

1. Aiello AE, Larson EL, Sedlak R. Personal health. Bringing good hygiene home. Am J Infect Control. 2008;36:152-65.

2. Holt GR. Importance of personal hygiene techniques in public health. South Med J. 2012;105:5.

3. Bland A, Mawson L, Burden S. Developing healthcare skills through simulation. London: SAGE Publications; 2012.

4. UK Department of Health. Essence of care: benchmarks for personal hygiene 2010. Available from: https://www.gov.uk/ government/uploads/system/uploads/attachment_data/file/153464/ dh_119976.pdf.pdf.

5. Frey Rebecca J. The Gale encyclopedia of senior health: a guide for seniors and their caregivers. Detroit: Gale; 2009.

6. Liao Q, Cowling BJ, Lam WW, Fielding R. The influence of social-cognitive factors on personal hygiene practices to protect against influenzas: using modelling to compare avian A/H5N1 and 2009 pandemic A/H1N1 influenzas in Hong Kong. Int J Behav Med. 2011;18:93-104.

7. Griffiths J. Meeting personal hygiene needs in the community: a district nursing perspective on the health and social care divide. Health Soc Care Community. 1998;6:234-40.

8. Weyant R. Interventions based on psychological principles improve adherence to oral hygiene instructions. J Evid Based Dent Pract. 2009;9:9-10.

9. Newton JT. Psychological models of behaviour change and oral hygiene behaviour in individuals with periodontitis: a call for more and better trials of interventions. J Clin Periodontol. 2010;37:910-1.

10. Parker L. Infection control: maintaining the personal hygiene of patients and staff. Br J Nurs. 2004;13:474-8.

11. Bloomfield SF. Home hygiene: a risk approach. Int J Hyg Environ Health. 2003;206:1-8.

12. Aiello AE, Coulborn RM, Perez V, Larson EL. Effect of hand hygiene on infectious disease risk in the community setting: a meta-analysis. Am J Public Health. 2008;98:1372-81.

13. Miko BA, Cohen B, Conway L, Gilman A, Seward SL, Larson E. Determinants of personal and household hygiene among college students in New York City, 2011. Am J Infect Control. 2012;40:940-5.

14. Mbawalla HS, Masalu JR, Astrom AN. Socio-demographic and behavioural correlates of oral hygiene status and oral health related quality of life, the Limpopo-Arusha school health project (LASH): A cross-sectional study. BMC Pediatr 2010; 10.

15. Barretto EPR, Pordeus IA, Ferreira EF, Paiva SM. Quality of infantile life: influence of the habits of oral hygiene and of the access to the dental services. J Dental Res. 2003;82:205.

16. Fewtrell L, Kaufmann RB, Kay D, Enanoria W, Haller L, Colford JM. Water, sanitation, and hygiene interventions to reduce diarrhoea in less developed countries: a systematic review and metaanalysis. Lancet Infect Dis. 2005;5:42-52.

17. Turabelidze G, Lin M, Wolkoff B, Dodson D, Gladbach S, Zhu BP. Personal hygiene and methicillin-resistant Staphylococcus aureus infection. Emerg Infect Dis. 2006;12:422-7.

18. Timmer K, Bock E, Tumena T. Personal hygiene assessmentsimple instrument for the assessment of personal hygiene. Z Gerontol Geriatr. 2004;37:51.

19. Burford B, Hesketh A, Wakeling J, Bagnall G, Colthart I, Illing J, et al. Asking the right questions and getting meaningful responses: 12 tips on developing and administering a questionnaire survey for healthcare professionals. Med Teach. 2009;31:207-11.

20. Wardell DW, Czerwinski B. A military challenge to managing feminine and personal hygiene. J Am Acad Nurse Pract. 2001;13:187-93. 
21. Linton DS. "War dysentery" and the limitations of German military hygiene during World War I. Bull Hist Med. 2010;84:607-39.

22. Surgeoner BV, Chapman BJ, Powell DA. University students' hand hygiene practice during a gastrointestinal outbreak in residence: what they say they do and what they actually do. J Environ Health. 2009;72:24-8.

23. Cowdell F. Older people, personal hygiene, and skin care. Medsurg Nurs. 2011;20:235-40.

24. [No authors listed]. Dental classification and risk assessment prevention of dental morbidity in deployed military personnel. Proceedings of an international workshop. Mil Med. 2008; 173:1-59.

25. German V, Kopterides P, Poulikakos P, Giannakos G, Falagas ME. Respiratory tract infections in a military recruit setting: a prospective cohort study. J Infect Public Health. 2008;1:101-4.

26. Van Camp RO, Ortega HJ. Hand sanitizer and rates of acute illness in military aviation personnel. Aviat Space Environ Med. 2007;78:140-2.

27. Emami A, Momeni P, Hossein MA, Maddah SS. Developing a questionnaire for conducting cross-national studies-'Self-reported health and needs among elderly Iranians and Swedes'. Scand J Caring Sci. 2010;24:372-9.

28. Stevenson RJ, Case TI, Hodgson D, Porzig-Drummond R, Barouei J, Oaten MJ. A scale for measuring hygiene behavior: development, reliability and validity. Am J Infect Control. 2009;37:557-64.

29. Webb AL, Stein AD, Ramakrishnan U, Hertzberg VS, Urizar M, Martorell R. A simple index to measure hygiene behaviours. Int J Epidemiol. 2006;35:1469-77.
30. Anastasi A, Urbina S. Psychological testing. 7th edn. Upper Saddle River: Prentice Hall; 1997.

31. Polgar S, Thomas SA. Introduction to research in the health sciences, 5th edn. Edinburgh: Elsevier; 2008.

32. Cottrell RR, McKenzie JF. Health promotion and education research methods: using the five-chapter thesis/dissertation model, 2nd edn. Sudbury: Jones and Bartlett Publishers; 2011.

33. Heilman CB. Readability of patient education materials. World Neurosurg. 2011 [Epub ahead of print].

34. World Health Organization. Personal, domestic and community hygiene (database on the Internet); 2010. Available from: http:// www.who.int/water_sanitation_health/hygiene/settings/hvchap8. pdf. Accessed 13 May 2013

35. Sakharova OB, Kiku PF, Gorborukova TV. The impact of social and hygienic lifestyle factors on health status of students. Gig Sanit. 2012:54-8.

36. Avshits IV, Shirinskii VA. Health status hygienic assessment of primary military education establishment pupils. Gig Sanit. 2010:76-8.

37. Buunk-Werkhoven YA, Dijkstra A, van der Wal H, Basic N, Loomans SA, van der Schans CP, et al. Promoting oral hygiene behavior in recruits in the Dutch Army. Mil Med. 2009;174:971-6.

38. Mehrabi A, Esmi N, Khubdel M. Soldiers' knowledge on hygiene status in a military training center. J Mil Med. 2009;10:293-7.

39. Chalyi NN, Bazhenov VG. Experience with the health and hygiene and epidemic control support for the troops and the population under extreme situations (a review of the literature). Voen Med Zh. 1994:14-7. 